\title{
SOME REMARKS ON THE BILOCAL PROBLEM
}

\author{
OCTAVIA BOLOJAN AND SORIN MUREŞAN
}

Received 30 October, 2015

\begin{abstract}
Using a method proposed by T.A. Burton in [6] and Banach's Contraction Principle, we study the existence, uniqueness and approximation of the solution for a bilocal problem. We compare our results with the classical similarly results given for the Fredholm integral operator associated. The present paper extends and complements the results from [14].
\end{abstract}

2010 Mathematics Subject Classification: 35A35; 47G10; 47H10; 54H25

Keywords: bilocal problem, contraction principle, Fredholm integral operator, successive approximation sequence

\section{INTRODUCTION}

It is well-known (see $[1,3,9,20])$ that the existence, uniqueness and the approximation of the solution for the bilocal problem

$$
\left\{\begin{array}{l}
x^{\prime \prime}(t)=f\left(t, x(t), x^{\prime}(t)\right) \\
x(a)=x_{0}, x(b)=x_{1}
\end{array}, \quad t \in[a, b]\right.
$$

can be proved using the Contraction Principle for the Fredholm integral operator

$$
\begin{gathered}
F: C^{1}[a, b] \rightarrow C^{1}[a, b], \\
F(x)(t)=\frac{b-t}{b-a} x_{0}+\frac{t-a}{b-a} x_{1}-\int_{a}^{b} G(t, s) f\left(s, x(s), x^{\prime}(s)\right) d s,
\end{gathered}
$$

where

$$
G(t, s)= \begin{cases}\frac{(b-t)(s-a)}{b-a}, & \text { if } a \leq s \leq t \\ \frac{(t-a)(b-s)}{b-a}, & \text { if } t \leq s \leq b\end{cases}
$$

The first author was supported by the Sectorial Operational Programme for Human Resources Development 2007-2013, co-financed by the European Social Fund, under the project POSDRU/159/1.5/S/137750, by a grant of the Romanian National Authority for Scientific Research, CNCSUEFISCDI, project number PN-II-ID-PCE-2011-3-0094 and by a grant of the Babeş-Bolyai University of Cluj-Napoca, through GTC-2016, project number 31782, project title "Operatorial analysis techniques for nonlinear systems with nonlocal conditions and applications". 
is the corresponding Green function. Therefore, a function $x \in C^{1}[a, b]$ is a solution of (1.1) if $x(t)$ is a fixed point of the Fredholm integral operator (1.2) (see [1,9,18, 21,24]).

In the last thirty years, it has been given a particular interest in studying the properties of the solutions for a large class of problems from nonlinear analysis. For example, the study of the periodic solutions of two-point boundary value problems using the method of successive approximations has been made in [19] and [22]. Moreover, in [17], Precup studies the existence, uniqueness and iterative approximation of solutions to a boundary value problem for second-order differential equation on bounded sets in Banach spaces. The tools used here are an extension of Granas' continuation principle for contractive mappings to spaces endowed with two metrics. Other existence results that were obtained for these type of problems can be found in [16]. Then, in [12] the problem of existence of solutions for Fredholm and Volterra integral equations are investigated. Using a monotone method, are given sufficient conditions when integral equations have their solutions in a corresponding sector. Here, some delay differential and integral inequalities are also discussed. Returning to the method of successive approximations, recently, in [5], are presented some convergence and numerical stability properties of the method applied to two-point boundary value problems for nonlinear second order neutral differential equations in Banach spaces. The considered algorithm has a practical stopping criterion and its accuracy is illustrated by examples. Other discussions about the properties of the successive approximation sequence and the numerical methods built using them can be found in $[2,4,7,8,10,11,13,23]$.

Following a method proposed by T.A. Burton in [6], in this paper we study the bilocal problem using the Contraction Principle for another operator, namely:

$$
\begin{aligned}
B: C[a, b] \rightarrow C[a, b], & \\
B(u)(t) & =f\left(t, \frac{b-t}{b-a} x_{0}+\frac{t-a}{b-a} x_{1}-\int_{a}^{b} G(t, s) u(s) d s,\right. \\
& \left.\frac{x_{1}-x_{0}}{b-a}-\int_{a}^{b} H(t, s) u(s) d s\right),
\end{aligned}
$$

where

$$
H(t, s)=\left\{\begin{array}{ll}
\frac{a-s}{b-a}, & \text { if } a \leq s \leq t \\
\frac{b-s}{b-a}, & \text { if } t \leq s \leq b
\end{array} .\right.
$$

More exactly, we construct an approximation scheme for the solution of the bilocal problem (1.1) based on the method of successive approximations. Since the constant obtained for the so-called Burton operator defined by (1.4) is less than the constant 
of the Fredholm operator, the successive approximations sequence offers a better approximation for the solution of problem (1.1).

Burton's approach was also exemplified in [14] and the main purpose was to approximate the solution of the Cauchy problem and its derivative using two operators: Volterra operator, in the classical case, and Burton operator, after the method proposed in [6]. Then, in [15], the same method was used to give existence and uniqueness results for first order differential equations and systems.

Throughout the paper, the norms on $C^{1}[a, b]$ and $C[a, b]$ are given by

$$
\|x\|^{*}=\sup _{t \in[a, b]}|x(t)|+\sup _{t \in[a, b]}\left|x^{\prime}(t)\right|
$$

and

$$
\|u\|=\sup _{t \in[a, b]}|u(t)|
$$

respectively.

We also recall an important existence and uniqueness result that we shall use in our framework (see [1]). Here, the solution of the bilocal problem is given as the fixed point of the Fredholm operator (1.2), where the considered norm is given by (1.6).

Theorem 1. If there exist two positive constants $L_{1}$ and $L_{2}$ such that the function $f:[a, b] \times \mathbb{R} \times \mathbb{R} \rightarrow \mathbb{R}$ satisfy the Lipschitz condition

$$
|f(t, u, v)-f(t, \bar{u}, \bar{v})| \leq L_{1}|u-\bar{u}|+L_{2}|v-\bar{v}|,
$$

for all $t \in[a, b], u, v, \bar{u}, \bar{v} \in \mathbb{R}$, and

$$
\alpha_{F}=\max \left\{L_{1}, L_{2}\right\}\left(\frac{(b-a)^{2}}{8}+\frac{b-a}{2}\right)<1
$$

holds, then:

(i) problem (1.1) has a unique solution $x^{*}$, the unique fixed point of the Fredholm operator (1.2);

(ii) $x^{*}$ can be approximated by the sequence $x_{0}, x_{1}=F\left(x_{0}\right), x_{2}=F\left(x_{1}\right), \ldots, x_{n}=$ $F\left(x_{n-1}\right), \ldots$ and we have

$$
\left\|x_{n}-x^{*}\right\|^{*} \leq \frac{\alpha_{F}^{n}}{1-\alpha_{F}}\left\|x_{1}-x_{0}\right\|^{*}
$$

for all $n \geq 1$.

The paper is organized as follows: in Section 2 using Burton's method, we obtain an existence and uniqueness results for the solution of the bilocal problem (1.1) and then, in Section 3 we present the main results in approximating this solution with the sequence of successive approximations of the two operators defined by (1.2) and (1.4). As we have already mentioned, since Banach's Contraction Principle offers an 
approximation of the fixed point, we compare these approximations in the last section.

\section{EXISTENCE AND UNIQUENESS FOR BURTON OPERATOR CASE}

In order to obtain the fixed point problem for the Burton operator (1.4), which is equivalent with the considered bilocal problem, we start with

$$
x^{\prime}(t)=\int_{a}^{t} x^{\prime \prime}(s) d s+x^{\prime}(a),
$$

which gives

$$
\begin{aligned}
x(t) & =\int_{a}^{t} x^{\prime}(s) d s+x(a) \\
& =\int_{a}^{t}\left(\int_{a}^{s} x^{\prime \prime}(u) d u\right) d s+x^{\prime}(a)(t-a)+x_{0} \\
& =\int_{a}^{t} \int_{u}^{t} x^{\prime \prime}(u) d s d u+x^{\prime}(a)(t-a)+x_{0} \\
& =\int_{a}^{t}(t-s) x^{\prime \prime}(s) d s+x^{\prime}(a)(t-a)+x_{0} .
\end{aligned}
$$

Since $x_{1}=x(b)$ we have that

$$
x_{1}=\int_{a}^{b}(b-s) x^{\prime \prime}(s) d s+x^{\prime}(a)(b-a)+x_{0},
$$

obtaining the formula for $x^{\prime}(a)$,

$$
x^{\prime}(a)=\frac{x_{1}-x_{0}}{b-a}-\int_{a}^{b} \frac{b-s}{b-a} x^{\prime \prime}(s) d s .
$$

Using this formula, we obtain that

$$
x^{\prime}(t)=\frac{x_{1}-x_{0}}{b-a}-\int_{a}^{b} H(t, s) x^{\prime \prime}(s) d s
$$


and

$$
x(t)=\frac{b-t}{b-a} x_{0}+\frac{t-a}{b-a} x_{1}-\int_{a}^{b} G(t, s) x^{\prime \prime}(s) d s,
$$

where $G(t, s), H(t, s)$ are given by (1.3) and (1.5), respectively.

Replacing (2.1) and (2.2) in (1.1), the bilocal problem (1.1) is equivalent with

$$
\begin{aligned}
x^{\prime \prime}(t) & =f\left(t, \frac{b-t}{b-a} x_{0}+\frac{t-a}{b-a} x_{1}-\int_{a}^{b} G(t, s) x^{\prime \prime}(s) d s,\right. \\
& \left.\frac{x_{1}-x_{0}}{b-a}-\int_{a}^{b} H(t, s) x^{\prime \prime}(s) d s\right) .
\end{aligned}
$$

At this point, Burton's approach suggests to find solutions of (1.1) by using the fixed points of the operator (1.4). This appears if problem (1.1) is rewritten as

$$
\begin{aligned}
x^{\prime \prime}(t) & =f\left(t, \frac{b-t}{b-a} x_{0}+\frac{t-a}{b-a} x_{1}-\int_{a}^{b} G(t, s) x^{\prime \prime}(s) d s,\right. \\
& \left.\frac{x_{1}-x_{0}}{b-a}-\int_{a}^{b} H(t, s) x^{\prime \prime}(s) d s\right) \equiv B\left(x^{\prime \prime}\right)(t) .
\end{aligned}
$$

In order to obtain the equivalent integral form of the bilocal problem, denote

$$
x^{\prime \prime}(t)=u(t) .
$$

Hence, the solution of the bilocal problem is the solution of the Burton operator defined by (1.4).

In what follows, we give our main existence and uniqueness result. For this, we consider the problem (1.4) defined on the Banach space $C[a, b]$, with the norm given by (1.7).

Theorem 2. If function $f:[a, b] \times \mathbb{R} \times \mathbb{R} \rightarrow \mathbb{R}$ satisfy the Lipschitz condition (1.8) and

$$
\alpha_{B}=L_{1} \cdot \frac{(b-a)^{2}}{8}+L_{2} \cdot \frac{b-a}{2}<1
$$

holds, then:

(i) problem (1.1) has a unique solution $x^{*}$ which can be determined with $u^{*}$, the unique fixed point of the Burton operator (1.4);

(ii) $u^{*}$ can be approximated by the sequence $u_{0}, u_{1}=B\left(u_{0}\right), u_{2}=B\left(u_{1}\right), \ldots, u_{n}=$ 
$B\left(u_{n-1}\right), \ldots$ and we have

$$
\left\|u_{n}-u^{*}\right\| \leq \frac{\alpha_{B}^{n}}{1-\alpha_{B}}\left\|u_{1}-u_{0}\right\|,
$$

for all $n \geq 1$.

Proof. (i) For any $u, \bar{u} \in C[a, b]$ we have

$$
\begin{aligned}
& |B(u)(t)-B(\bar{u})(t)| \\
& \leq L_{1} \cdot \int_{a}^{b}|G(t, s)||u(s)-\bar{u}(s)| d s+L_{2} \cdot \int_{a}^{b}|H(t, s)||u(s)-\bar{u}(s)| d s \\
& \leq\left(L_{1} \cdot \sup \int_{a}^{b}|G(t, s)| d s+L_{2} \cdot \sup \int_{a}^{b}|H(t, s)| d s\right)\|u-\bar{u}\| \\
& \leq\left(L_{1} \cdot \frac{(b-a)^{2}}{8}+L_{2} \cdot \frac{b-a}{2}\right)\|u-\bar{u}\| .
\end{aligned}
$$

Taking the supremum, we have that

$$
\|B(u)(t)-B(\bar{u})(t)\| \leq \alpha_{B} \cdot\|u-\bar{u}\| .
$$

Since $\alpha_{B}<1$ we have that the operator $B$ is a $\alpha_{B}$-contraction and hence, the existence and uniqueness of the fixed point of (1.4). Now, applying Banach's Contraction Principle, we obtain $(i$ i $)$. Moreover, the unique solution of the considered bilocal problem (1.1) is given by

$$
x^{*}(t)=\frac{b-t}{b-a} x_{0}+\frac{t-a}{b-a} x_{1}-\int_{a}^{b} G(t, s) u^{*}(s) d s,
$$

where $G(t, s)$ is the corresponding Green function.

\section{COMPARISON OF THE APPROXIMATIONS}

In the previous sections we have given two existence and uniqueness results for the solution of the bilocal problem (1.1). But, in the classical result given in Theorem 1 we have that the fixed point of the Fredholm operator (1.2) is the solution $x^{*}(t)$ for the "trajectory" $x(t)$; also, from Theorem 2, the fixed point of the Burton operator (1.4) is the solution $u^{*}(t)$ for the "acceleration" $x^{\prime \prime}(t)$. In both cases we have the approximation of the solution with the successive approximations sequences $\left(F\left(x_{n}\right)\right)_{n \in \mathbb{N}}$ and $\left(B\left(u_{n}\right)\right)_{n \in \mathbb{N}}$, respectively. In what follows we study in what manner we can approximate $x^{*}(t)$ by the sequence $\left(B\left(u_{n}\right)\right)_{n \in \mathbb{N}}$ and also, $u^{*}(t)$ by the sequence $\left(F\left(x_{n}\right)\right)_{n \in \mathbb{N}}$. 
Proposition 1. If function $f:[a, b] \times \mathbb{R} \times \mathbb{R} \rightarrow \mathbb{R}$ satisfy the Lipschitz condition (1.8) and there exist some constants $\alpha_{F}$ given by (1.9) and $\alpha_{B}$ given by (2.3) such that $\alpha_{B} \leq \alpha_{F}$, then:

(i) For any $x_{0} \in C^{1}[a, b]$, the sequence $\left(F\left(x_{n}\right)\right)_{n \in \mathbb{N}}$ has the property

$$
\left\|\left(F\left(x_{n}\right)\right)^{\prime \prime}-u^{*}\right\| \leq\left(\alpha_{B}\right)^{n-1}\left\|\left(F\left(x_{0}\right)\right)^{\prime \prime}-u^{*}\right\| ;
$$

(ii) For any $u_{0} \in C[a, b]$, the sequence $\left(B\left(u_{n}\right)\right)_{n \in \mathbb{N}}$ has the property

$$
\left\|B\left(u_{n}\right)-x^{*}\right\|^{*} \leq\left(\alpha_{F}\right)^{n-1}\left\|B\left(u_{0}\right)-x^{*}\right\|^{*} .
$$

Proof. First, we observe that

$$
\begin{aligned}
& \left(x^{*}\right)^{\prime \prime}=u^{*}, \\
& x^{*}(t)=\frac{b-t}{b-a} x_{0}+\frac{t-a}{b-a} x_{1}-\int_{a}^{b} G(t, s) u^{*}(s) d s,
\end{aligned}
$$

and, since $\alpha_{B} \leq \alpha_{F}$, we obtain that $\alpha_{B}<1$. Hence, both Theorems 2 and 1 can be applied.

(i) Consider the sequence $\left(x_{n}\right)_{n \in \mathbb{N}}$ with

$$
\begin{aligned}
x_{n}(t) & =F\left(x_{n-1}\right)(t)= \\
& =\frac{b-t}{b-a} x_{0}+\frac{t-a}{b-a} x_{1}-\int_{a}^{b} G(t, s) f\left(s, x_{n-1}(s), x_{n-1}^{\prime}(s)\right) d s, \\
x_{n}^{\prime}(t) & =\frac{-1}{b-a} x_{0}+\frac{1}{b-a} x_{1}-\int_{a}^{b} H(t, s) f\left(s, x_{n-1}(s), x_{n-1}^{\prime}(s)\right) d s
\end{aligned}
$$

and

$$
x_{n}^{\prime \prime}(t)=f\left(t, x_{n-1}(t), x_{n-1}^{\prime}(t)\right) .
$$

We have that

$$
\begin{aligned}
& \left|x_{n}^{\prime \prime}(t)-u^{*}(t)\right| \\
& =\mid f\left(t, x_{n-1}(t), x_{n-1}^{\prime}(t)\right)- \\
& -f\left(t, \frac{b-t}{b-a} x_{0}+\frac{t-a}{b-a} x_{1}-\int_{a}^{b} G(t, s) u^{*}(s) d s,\right. \\
& \left.\frac{-1}{b-a} x_{0}+\frac{1}{b-a} x_{1}-\int_{a}^{b} H(t, s) u^{*}(s) d s\right)
\end{aligned}
$$




$$
\begin{aligned}
& \leq L_{1}\left|x_{n-1}(t)-\frac{b-t}{b-a} x_{0}-\frac{t-a}{b-a} x_{1}+\int_{a}^{b} G(t, s) u^{*}(s) d s\right| \\
& +L_{2}\left|x_{n-1}^{\prime}(t)-\frac{x_{1}-x_{0}}{b-a}+\int_{a}^{b} H(t, s) u^{*}(s) d s\right| \\
& \leq L_{1} \int_{a}^{b}|G(t, s)| \cdot\left|x_{n-1}^{\prime \prime}(s)-u^{*}(s)\right| d s \\
& +L_{2} \int_{a}^{b}|H(t, s)| \cdot\left|x_{n-1}^{\prime \prime}(s)-u^{*}(s)\right| d s \\
& \leq\left(L_{1} \cdot \sup \int_{a}^{b}|G(t, s)| d s+L_{2} \cdot \sup \int_{a}^{b}|H(t, s)| d s\right)\left\|x_{n-1}^{\prime \prime}(s)-u^{*}(s)\right\| \\
& \leq\left(L_{1} \cdot \frac{(b-a)^{2}}{8}+L_{2} \cdot \frac{b-a}{2}\right)\left\|x_{n-1}^{\prime \prime}(s)-u^{*}(s)\right\| .
\end{aligned}
$$

From the calculus above, we conclude that the aproximation for $u^{*}$ is given by:

$$
\left\|x_{n}^{\prime \prime}-u^{*}\right\| \leq\left(\alpha_{B}\right)^{n-1}\left\|x_{1}^{\prime \prime}-u^{*}\right\| \text {. }
$$

(ii) Considering

$$
\begin{aligned}
& y_{n}(t)=B\left(u_{n}\right)(t)=\frac{b-t}{b-a} x_{0}+\frac{t-a}{b-a} x_{1}-\int_{a}^{b} G(t, s) u_{n}(s) d s, \\
& y_{n}^{\prime}(t)=\frac{x_{1}-x_{0}}{b-a}+\int_{a}^{b} H(t, s) u_{n}(s) d s
\end{aligned}
$$

and

$$
\begin{gathered}
x^{*}(t)=\frac{b-t}{b-a} x_{0}+\frac{t-a}{b-a} x_{1}-\int_{a}^{b} G(t, s) f\left(s, x^{*}(s),\left(x^{*}\right)^{\prime}(s)\right) d s, \\
\left(x^{*}\right)^{\prime}(t)=\frac{x_{1}-x_{0}}{b-a}+\int_{a}^{b} H(t, s) f\left(s, x^{*}(s),\left(x^{*}\right)^{\prime}(s)\right) d s,
\end{gathered}
$$

respectively, we obtain

$$
\left|y_{n}(t)-x^{*}(t)\right|
$$




$$
\begin{aligned}
& \mid \frac{b-t}{b-a} x_{0}+\frac{t-a}{b-a} x_{1}-\int_{a}^{b} G(t, s) u_{n}(s) d s \\
- & \frac{b-t}{b-a} x_{0}-\frac{t-a}{b-a} x_{1}+\int_{a}^{b} G(t, s) f\left(s, x^{*}(s),\left(x^{*}\right)^{\prime}(s)\right) d s \mid \\
\leq & \int_{a}^{b}|G(t, s)| \cdot\left|u_{n}(s)-f\left(s, x^{*}(s),\left(x^{*}\right)^{\prime}(s)\right)\right| d s \\
= & \int_{a}^{b}|G(t, s)| \cdot \mid f\left(s, \frac{b-s}{b-a} x_{0}+\frac{s-a}{b-a} x_{1}-\int_{a}^{b} G(t, s) u_{n-1}(s) d s,\right. \\
& \left.\frac{x_{1}-x_{0}}{b-a}-\int_{a} H(t, s) u_{n-1}(s)\right)-f\left(s, x^{*}(s),\left(x^{*}\right)^{\prime}(s)\right) \mid d s \\
& \leq \int_{a}|G(t, s)| L_{1} \cdot\left|y_{n-1}(s)-x^{*}(s)\right|+L_{2} \cdot\left|y_{n-1}^{\prime}(s)-\left(x^{*}\right)^{\prime}(s)\right| d s \\
\leq & \max \left\{L_{1}, L_{2}\right\} \cdot \sup \int_{a}^{b}|G(t, s)| d s\left\|y_{n-1}-x^{*}\right\|^{*} \\
= & \max \left\{L_{1}, L_{2}\right\} \cdot \frac{(b-a)^{2}}{8} \cdot\left\|y_{n-1}-x^{*}\right\|^{*} .
\end{aligned}
$$

We also have that

$$
\begin{aligned}
& \left|y_{n}^{\prime}(t)-\left(x^{*}\right)^{\prime}(t)\right| \\
& =\left|\frac{x_{1}-x_{0}}{b-a}-\int_{a}^{b} H(t, s) u_{n}(s) d s-\frac{x_{1}-x_{0}}{b-a}+\int_{a}^{b} H(t, s) f\left(s, x^{*}(s),\left(x^{*}\right)^{\prime}(s)\right) d s\right| \\
& \leq \int_{a}^{b}|H(t, s)| \cdot\left|u_{n}(s)-f\left(s, x^{*}(s),\left(x^{*}\right)^{\prime}(s)\right)\right| d s \\
& \quad=\int_{a}^{b}|H(t, s)| \cdot\left|f\left(s, y_{n-1}(s), y_{n-1}^{\prime}(s)\right)-f\left(s, x^{*}(s),\left(x^{*}\right)^{\prime}(s)\right)\right| d s
\end{aligned}
$$




$$
\begin{aligned}
& \leq \int_{a}^{b}|H(t, s)|\left(L_{1} \cdot\left|y_{n-1}(s)-x^{*}(s)\right|+L_{2} \cdot\left|y_{n-1}^{\prime}(s)-\left(x^{*}\right)^{\prime}(s)\right|\right) d s \\
& \leq \max \left\{L_{1}, L_{2}\right\} \cdot \sup \int_{a}^{b}|G(t, s)| d s\left\|y_{n}-x^{*}\right\| \\
& =\max \left\{L_{1}, L_{2}\right\} \cdot \frac{(b-a)^{2}}{8} \cdot\left\|y_{n}-x^{*}\right\| .
\end{aligned}
$$

Hence, we conclude that the aproximation for $x^{*}$ is given by

$$
\left\|y_{n}-x^{*}\right\|^{*} \leq\left(\alpha_{F}\right)^{n-1}\left\|y_{1}-x^{*}\right\|^{*} \text {. }
$$

The novelty of this paper is given by the results obtained in approximating the solutions of problem (1.1) using the two operators defined on different spaces and with different norms. A similar work was done in paper [14], but for the Cauchy problem.

\section{ACKNOWLEDGEMENTS}

The authors would like to warmly thank Professor T.A. Burton for the constructive advices and encouragements on continuing his approach for the method of successive approximations.

\section{REFERENCES}

[1] R. P. Agarwal, M. Meehan, and D. O'Regan, Fixed point theory and applications, ser. Cambridge Tracts in Mathematics. Cambridge: Cambridge University Press, 2001, vol. 141.

[2] Z. S. Athanassov, "Uniqueness and convergence of successive approximations for ordinary differential equations," Math. Japon., vol. 35, no. 2, pp. 351-367, 1990.

[3] D. F. Bailey, "Some theorems on contractive mappings," J. London Math. Soc., vol. 41, pp. 101106, 1966.

[4] A. Bellen and R. Vermiglio, "Some applications of continuous Runge-Kutta methods," Appl. Numer. Math., vol. 22, no. 1-3, pp. 63-80, 1996.

[5] A. M. Bica, "Properties of the method of successive approximations for two-point boundary value problems," J. Nonlinear Evol. Equ. Appl., vol. 2011, pp. 1-22, 2011.

[6] T. A. Burton, "Fixed points, differential equations, and proper mappings," Semin. Fixed Point Theory Cluj-Napoca, vol. 3, pp. 19-32, 2002.

[7] J. C. Butcher, "Numerical methods for ordinary differential equations in the 20th century," $J$. Comput. Appl. Math., vol. 125, no. 1-2, pp. 1-29, 2000.

[8] J. C. Butcher and A. D. Heard, "Stability of numerical methods for ordinary differential equations," Numer. Algorithms, vol. 31, no. 1-4, pp. 59-73, 2002.

[9] J. Dugundji and A. Granas, Fixed point theory. I, ser. Monografie Matematyczne [Mathematical Monographs]. Państwowe Wydawnictwo Naukowe (PWN - Polish Scientific Publishers), Warsaw, 1982, vol. 61. 
[10] A. Friedmann and A. Kharytonov, "Solving of nonlinear differential equations by a method of successive approximations," in Analysis, numerics and applications of differential and integral equations (Stuttgart, 1996), ser. Pitman Res. Notes Math. Ser. Longman, Harlow, 1998, vol. 379, pp. 97-100.

[11] T. E. Hull, W. H. Enright, B. M. Fellen, and A. E. Sedgwick, "Comparing numerical methods for ordinary differential equations," SIAM J. Numer. Anal., vol. 9, pp. 603-637, 1972.

[12] T. Jankowski, "Monotone method to Volterra and Fredholm integral equations with deviating arguments," Integral Transforms Spec. Funct., vol. 19, no. 1-2, pp. 95-104, 2008.

[13] P. Kama and E. A. Ibijola, "On a new one-step method for numerical solution of initial-value problems in ordinary differential equations," Int. J. Comput. Math., vol. 77, no. 3, pp. 457-467, 2001.

[14] S. Mureşan and O. Nica, "Comparing two integral operators in the approach of Cauchy problems," An. Univ. Oradea Fasc. Mat., vol. 19, no. 1, pp. 197-205, 2012.

[15] O. Nica, "A fixed point approach to first order differential equations and systems," Ann. Tiberiu Popoviciu Semin. Funct. Equ. Approx. Convexity, vol. 10, pp. 141-153, 2012.

[16] R. Precup, "Existence theorems for nonlinear problems by continuation methods," in Proceedings of the Second World Congress of Nonlinear Analysts, Part 6 (Athens, 1996), vol. 30, no. 6, 1997, pp. 3313-3322.

[17] R. Precup, "Discrete continuation method for boundary value problems on bounded sets in Banach spaces," J. Comput. Appl. Math., vol. 113, no. 1-2, pp. 267-281, 2000, fixed point theory with applications in nonlinear analysis.

[18] R. Precup, Methods in nonlinear integral equations. Kluwer Academic Publishers, Dordrecht, 2002.

[19] A. Rontó and M. Rontó, "Successive approximation techniques in non-linear boundary value problems for ordinary differential equations," in Handbook of differential equations: ordinary differential equations. Vol. IV, ser. Handb. Differ. Equ. Elsevier/North-Holland, Amsterdam, 2008, pp. 441-592.

[20] I. A. Rus, Differential equations, integral equations and dynamical systems (Ecuaţii diferenţiale, ecuaţii integrale şi sisteme dinamice - in Romanian). Transilvania Press, Cluj-Napoca, 1996.

[21] I. A. Rus, Generalized contractions and applications. Cluj University Press, Cluj-Napoca, 2001.

[22] A. M. Samoilenko and N. I. Ronto, "Modification of the numerical-analytical method of successive approximations for boundary value problems of ordinary differential equations," Ukrain. Mat. Zh., vol. 42, no. 8, pp. 1107-1116, 1990.

[23] L. F. Shampine, Numerical solution of ordinary differential equations. Chapman \& Hall, New York, 1994.

[24] E. Zeidler, Nonlinear functional analysis and its applications. II/B. Springer-Verlag, New York, 1990.

Authors' addresses

Octavia Bolojan

Babeş-Bolyai University, Department of Mathematics,, 400084, Cluj-Napoca, Romania

E-mail address: octavia.nica@math.ubbcluj.ro

\section{Sorin Mureşan}

University of Oradea, Department of Mathematics,, 410087, Oradea, Romania

E-mail address: smuresan@uoradea.ro 